\title{
Design of a Multiplexing Array for 3D Cardiac Imaging
}

\author{
Carolina Vallecilla, Jan D’hooge \\ Cardiovascular Imaging and Dynamics, Dept. of Cardiovascular Sciences, University of Leuven \\ Leuven, Belgium
}

\begin{abstract}
Volumetric cardiac ultrasound imaging requires $2 D$ matrix array transducers with thousands of elements to allow beam steering and focusing. Controlling all array elements independently is favorable but is expensive and impractical, especially for research purposes. One solution to this is the use of sparse transducers arrays in which not all transducer elements are effectively used. Alternatively, multiplexing (MUX'ing) could offer a solution by grouping various elements in a single electronic channel, reducing the channel count but keeping the array density. However, MUX'ed elements intrinsically share phase and amplitude when transmitting thereby impacting beam forming. Which transducer elements to be grouped into a single electronic channel is thus not obvious.

In this paper, a framework to find an optimal MUX configuration for a $3 D$ ultrasound system is presented using two different approaches: The matching algorithm and The virtual array. The two-way beam profiles were simulated as well as for a fully-wired array as a bench mark. To quantify these beam profiles, the side-lobe-tomain-lobe energy ratio (SMER) was computed for a beam focused along the diagonal in the $3 D$ image sequence (focus $60 \mathrm{~mm}$, opening angle of $37.5^{\circ}$ ). The frameworks have been applied to optimize a 32x32 element phased array transducer combined with a 256-channel front-end using a 1:4 MUX for cardiac imaging.

The mean SMER was $-33 \pm 2 d B$ for the fully wired array, $-28 \pm 5 d B$ for virtual array and $-23 \pm 3 d B$ for the matching algorithm array. The proposed MUX'ed probes are all suitable solutions for experimental $3 D$ cardiac ultrasound transducers. Although the virtual array shows the best SMER of the MUX'ed solution, receiving with $1 / 4$ of the array could carry image quality problems, while the matched array uses most of the elements on transmit and on receive using all the energy in acquisition, giving more flexibility to the system.
\end{abstract}

\section{Introduction}

Three-dimensional cardiac ultrasound imaging using matrix array transducers has become feasible in recent years making use of matrices containing thousands of elements. Commercial volumetric cardiac ultrasound systems control these arrays through two-stage beam forming in order to keep the wiring and the number of electronic channels limited and economically viable. However, this implies that elements cannot directly and individually be addressed. Alternatively, in the research setting, a few systems were proposed that allow addressing each element of a dense 2D matrix array independently up to 1024 elements. These systems come with relatively thick cables and dense channel density making them clinically impractical and expensive.
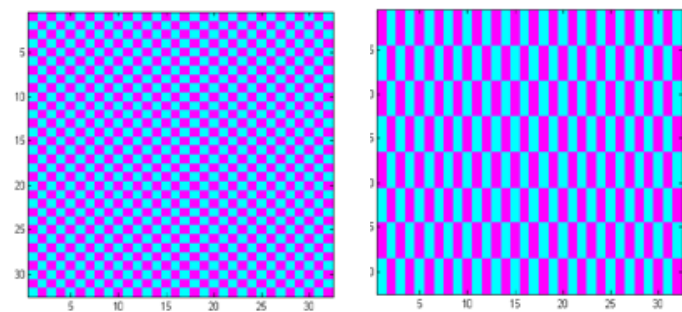

Fig 1. The virtual array. Elements from the fully wired array (left) are multiplexed in a vertical fashion creating a sort of "virtual array" (right).

Multiplexed (Mux) systems might offer an attractive alternative as they limit the number of electronic channels while keeping all transducer elements individually accessible. Although a MUXed system allows to control all array elements individually on transmit, it does not allow doing so in a fully independent manner thereby constraining the generated acoustic fields. Similarly, such systems allow to capture data from all elements by repeating the transmit event but this process can induce artifacts when imaging moving objects (i.e. cardiac imaging).

Designing a MUX-based system is challenging as one needs to decide which matrix array transducer elements will be multiplexed and thus connected to a single electronic transmit/receive channel. The aim of this work was therefore to develop a simulation framework to optimize the transmit multiplexing scheme for cardiac imaging. 
Virtual array Transmit
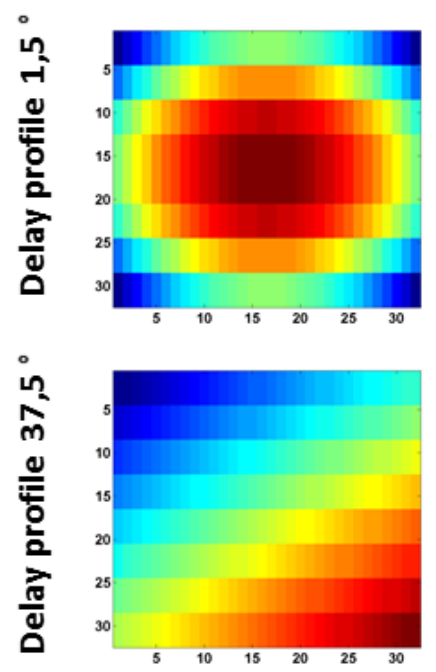

Virtual array Receive

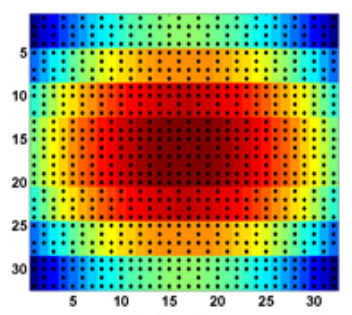

- black spots show

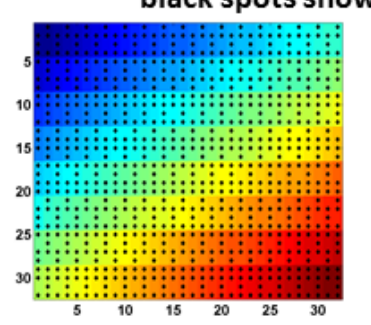

Match array Tx-Rx
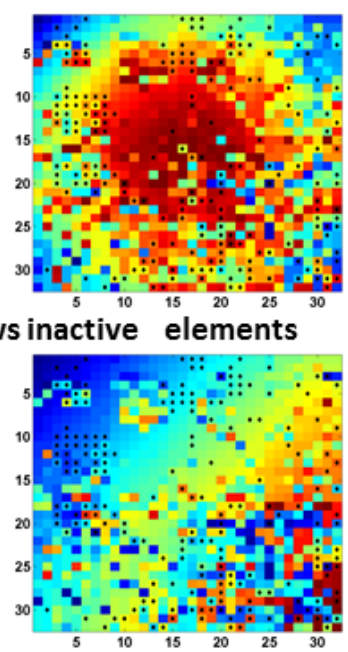

Fully wired array Tx-Rx

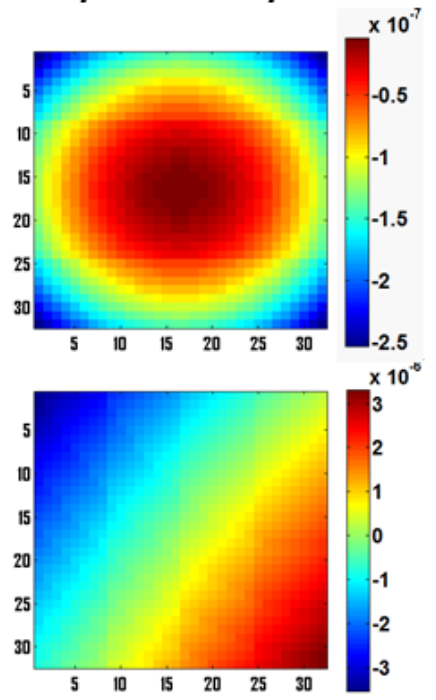

Fig 2. Delay profile for the two proposed approaches and for the fully wired array. Tx: transmit element configuration, Rx: receiving element configuration

\section{Methods}

We propose two methods to MUX 1024 elements (32x32 array) in 256 channels.

\subsection{The matching algorithm:}

In order to match $\mathrm{N}$ elements to $\mathrm{M}$ channels $(\mathrm{N}>\mathrm{M})$, an algorithm proposed in economics (Shapley 1962, Roth 1982) known as the theory of stable allocations and the practice of market design was used (e.g. assigning medical residents to hospitals where both parties can express preferences). The algorithm manages the excess of demand and attempts to give a "stable matching" where most of the requests are fulfilled. For our specific case, we applied the algorithm to assign elements (cf. residents) to channels (cf. hospitals). In particular, we tried to optimize the wiring of a 32x32-element array $(1 \times 1 \mathrm{~cm} 2)$ to a 256 -channel system using 1:4 on transmit and 4:1 on receive MUX'ing.

In order to express preferences of elements to belong to a certain channel, a 'co-occurrence' matrix (1024x1024) was built expressing the number of times each pair of elements transmits with a small (wrapped) phase mismatch (i.e. $<10 \%$ of the wave period) measured at the focal position (i.e. $60 \mathrm{~mm}$ ) for all transmit events (i.e. $50 \times 50$ ) in the image sequence (i.e. 3D imaging with an opening angle of $35.5^{\circ}$ ). The rationale for this being that MUX'ed elements will intrinsically transmit with the same phase. The 256 top-ranked 'co-occurrence' elements were assigned a channel each while the remaining elements could express their preference for a given channel based on their 'co-occurrence' value. When channels were preferred by more than 4 elements (i.e. excess of demand), priority was given to the ones with the highest co-occurrence value.

\subsection{The virtual array}

On this approach, elements were grouped geometrically by four consecutive elements creating a "virtual array" with bigger elements (4X height). The four MUX'ed elements in each channel were active on transmit but only one element was active on receive see Figure 1).

Both MUX'ed approaches were tested in Field II by simulating its two-way beam profile and contrasting it to that of the fully wired array. Side-lobe-to-main-lobeenergy-ratio (SMER) was calculated for both configurations while steering the beam in the azimuth and elevation directions as well as along the diagonal.

\section{Results and discussion}

Figure 2 shows the element delay disposition created after for the two approaches and figure 3 shows the two way beam profiles along the diagonal direction for the two proposed configuration and for the fully wired array. 
Virtual array

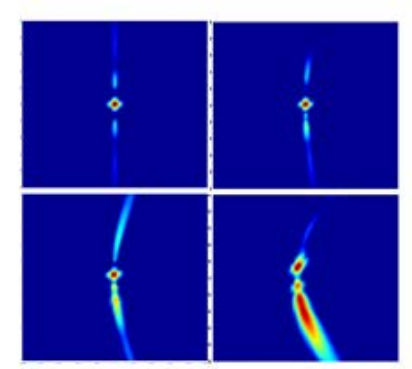

Match array

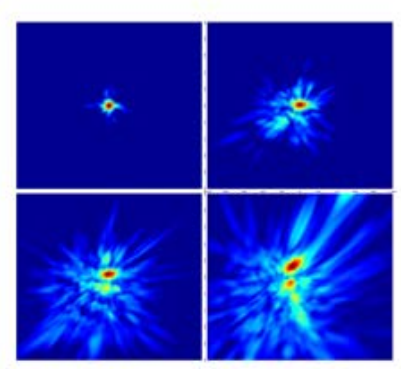

\section{Fully wired array}

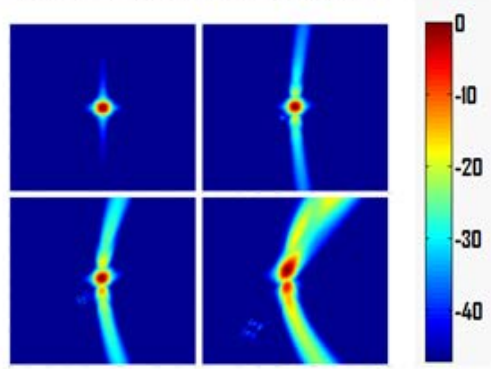

Fig 3. Two way beam profile for the two proposed approaches and for the fully wired array.

The mean SMER was $-33 \pm 2 \mathrm{~dB}$ for the fully wired array, $-28 \pm 5 \mathrm{~dB}$ for virtual array and $-23 \pm 3 \mathrm{~dB}$ for the matching algorithm array. SMER shows an acceptable energy distribution (below 22\%). Diagonal direction shows the highest SMER when steering out of 29 degrees for both arrays. The highest discrepancy occurs in azimuth beyond 30 degrees. Although the virtual array shows the best SMER of the MUX'ed solution, receiving with $1 / 4$ of the array could carry image quality problems, while the matched array uses most of the elements on transmit and on receive using all the energy in acquisition, giving more flexibility to the system.

\section{Conclusions}

The resulted MUXed probe is suitable for experimental 3D cardiac ultrasound transducers, where focused beams are required with low SMER. The proposed frame work could easily applied for other multiplexed-based systems where for example, the number of elements must be reduced.

\section{Acknowledgements}

The research leading to these results has received funding from the European Research Council under the European's Union Seventh Framework Program (FP7/2007-2013)/ERC Grant Agreement number 281748

We acknowledge Clodomiro Ferreira PhD (London Business School) for his collaboration on the matching algorithm.

Address for correspondence.

Carolina Vallecilla PhD

Università di Bologna, Department of Electrical, Electronic and Information Engineering. Viale Resorgimento 2, Bologna, Italy E-mail address: carolina.vallecilla@unibo.it 
